\title{
Zehn Jahre Vegetationsdynamik auf der Waldbrandfläche von Leuk (Wallis)
}

\author{
Thomas Wohlgemuth \\ Barbara Moser
}

Eidgenössische Forschungsanstalt für Wald, Schnee und Landschaft $(\mathrm{CH})^{*}$

Eidgenössische Forschungsanstalt für Wald, Schnee und Landschaft $(\mathrm{CH})$

\section{Zehn Jahre Vegetationsdynamik auf der Waldbrandfläche von Leuk (Wallis)}

\begin{abstract}
Der Waldbrand von Leuk (2003) erstreckte sich von 900 bis 2100 m ü. M. und war mit einer Fläche von 300 ha ein grosses Störungsereignis in der Schweiz, aber ein kleines im weltweiten Vergleich. Seine Lage entlang eines Höhengradienten bietet die einmalige Gelegenheit, die Entwicklung der Artenvielfalt und der natürlichen Verjüngung auf einer grossen Fläche in Abhängigkeit von verschiedenen Faktoren zu studieren. Während zehn Jahren wurde die Vegetationsentwicklung auf der Brandfläche in 148 Stichprobenflächen und fünf Zeitschnitten (2004, 2005, 2006, 2007 und 2013) verfolgt. Die Resultate zeigen, dass die Vielfalt der Pflanzenarten im vierten Aufnahmejahr am höchsten war und zu diesem Zeitpunkt auch deutlich die Vielfalt in den ungestörten Wäldern vor dem Feuer übertraf. Wo das Feuer intensiv brannte, entwickelte sich die Artenvielfalt während der ersten vier Jahre langsamer als an Orten mit geringerer Feuerintensität, und oberhalb von rund $1600 \mathrm{~m}$ ü.M. stieg die Artenvielfalt infolge der zahlreicher auftretenden Gebirgsarten deutlich an. In allen Höhenlagen schreitet die Wiederbewaldung voran. Die Anzahl Bäume mit mehr als $25 \mathrm{~cm}$ Höhe liegt zehn Jahre nach dem Feuer mit 1800 bis 3000 Stück pro Hektare eher auf einem niedrigen Niveau. Aspen (Populus tremula), Weiden (Salix appendiculata und S. caprea) sowie Birken (Betula pendula) sind am zahlreichsten, insbesondere in Höhenlagen bis 1700 m ü. M., wo die Verjüngung zu 95\% aus Laubbäumen besteht. Darüber wachsen Lärchen (Larix decidua) und Fichten (Picea abies) mit je 12\% Anteil an der Verjüngung nach. Im untersten Teil der Brandfläche etablieren sich nach zehn Jahren mehr Eichen (Quercus pubescens; 6.4\%) als Waldföhren (Pinus sylvestris; 3.7\%), doch sind die Anteile dieser ursprünglich bestandesbildenden Baumarten an der Gesamtstammzahl mit 10\% noch gering. In der Brandfläche entwickelt sich also ein Jungwald, aus dem im oberen Teil wieder ein Lärchen-Fichtenwald entsteht, während im unteren Teil ein Mischwald mit starker Beteiligung der Eiche aufwächst. Dort wird sich zeigen, ob und wie rasch die aktuell vorherrschenden Aspen, Weiden und Birken durch Nadelhölzer und Flaumeichen ersetzt werden.
\end{abstract}

Keywords: biodiversity, fire disturbance, resilience, tree regeneration, initial floristic composition Flora: Lauber et al (2012)

doi: $10.3188 /$ szf. 2018.0279

*Zürcherstrasse 111, CH-8903 Birmensdorf, E-Mail thomas.wohlgemuth@wsl.ch

A m Höhepunkt der extremen Hitzewelle 2003 brannte der Wald oberhalb von Leuk im August 2003 ab. Das von einem Brandstifter entfachte und sich in Windeseile ausbreitende Feuer hinterliess eine über $3 \mathrm{~km}$ lange und bis $\mathrm{zu} 1 \mathrm{~km}$ breite Schneise der Zerstörung. Bäume, Sträucher und die Bodenvegetation verbrannten auf einer Fläche von 300 ha, die von $900 \mathrm{~m}$ ü. M. bis an die Waldgrenze auf $2100 \mathrm{~m}$ ü. M. reichte. Das Ereignis löste die Ausarbeitung eines Waldbrandschutzkonzepts für das Wallis aus, verstärkte den Naturtourismus in der Region und motivierte viele Forschende für in- tensive und extensive Untersuchungen in diesem Gebiet. Heute, 15 Jahre nach dem grössten Waldbrand im Wallis der letzten 100 Jahre, ist ein Grossteil der Fläche von jungen Bäumen besiedelt, und Gras ist an vielen Orten über die Ascheschicht gewachsen. Dass hier eine derart grosse Brandfläche unbehandelt blieb und damit zu einem interessanten Beispiel der natürlichen Wiederbesiedlung und -bewaldung werden konnte, ist auf verschiedene politische Faktoren zurückzuführen, und letztlich als Glück für die Sukzessionsforschung zu bezeichnen. Die drängendste Fragen nach dem Ereignis waren, 
nach welchen Regeln die Wiederbesiedlung einer derart grossen Fläche abläuft und wie lange die Wiederbewaldung dauert (Wohlgemuth et al 2005).

In Regionen, wo Feuer ein natürliches Element der Vegetationsdynamik darstellt, haben sich Pflanzenarten in unterschiedlicher Weise an Brände angepasst, sei es durch Resistenz oder durch Regenerationsprozesse, die ohne Feuereinfluss nicht ablaufen würden (Gómez-González et al 2011, Keeley et al 2011). In diesen Regionen wurden typische Sukzessionsabläufe festgestellt, so in Australien (Gill 1997), im Mittelmeergebiet (Barbéro et al 1998), in der Borealzone (Johnstone \& Kasischke 2005) und auch im Tessin (Delarze et al 1992). Wo Feuer weniger häufig bzw. selten vorkommen oder erst im Rahmen von menschlichen Aktivitäten auftreten, sind die Folgen für die Vegetation bzw. die Abläufe der Wiederbesiedlung mit Pflanzen oft wenig bekannt und daher schwierig abzuschätzen. Zu diesen Gebieten zählen die Alpen, insbesondere das Wallis (Zumbrunnen et al 2009), Tirol und Kärnten (Vacik et al 2011) sowie inneralpine Täler in Frankreich (Magnier \& Trégouët 2011) und Italien (Vacchiano et al 2014). Langzeitbeobachtungen über die Vegetationsentwicklung auf

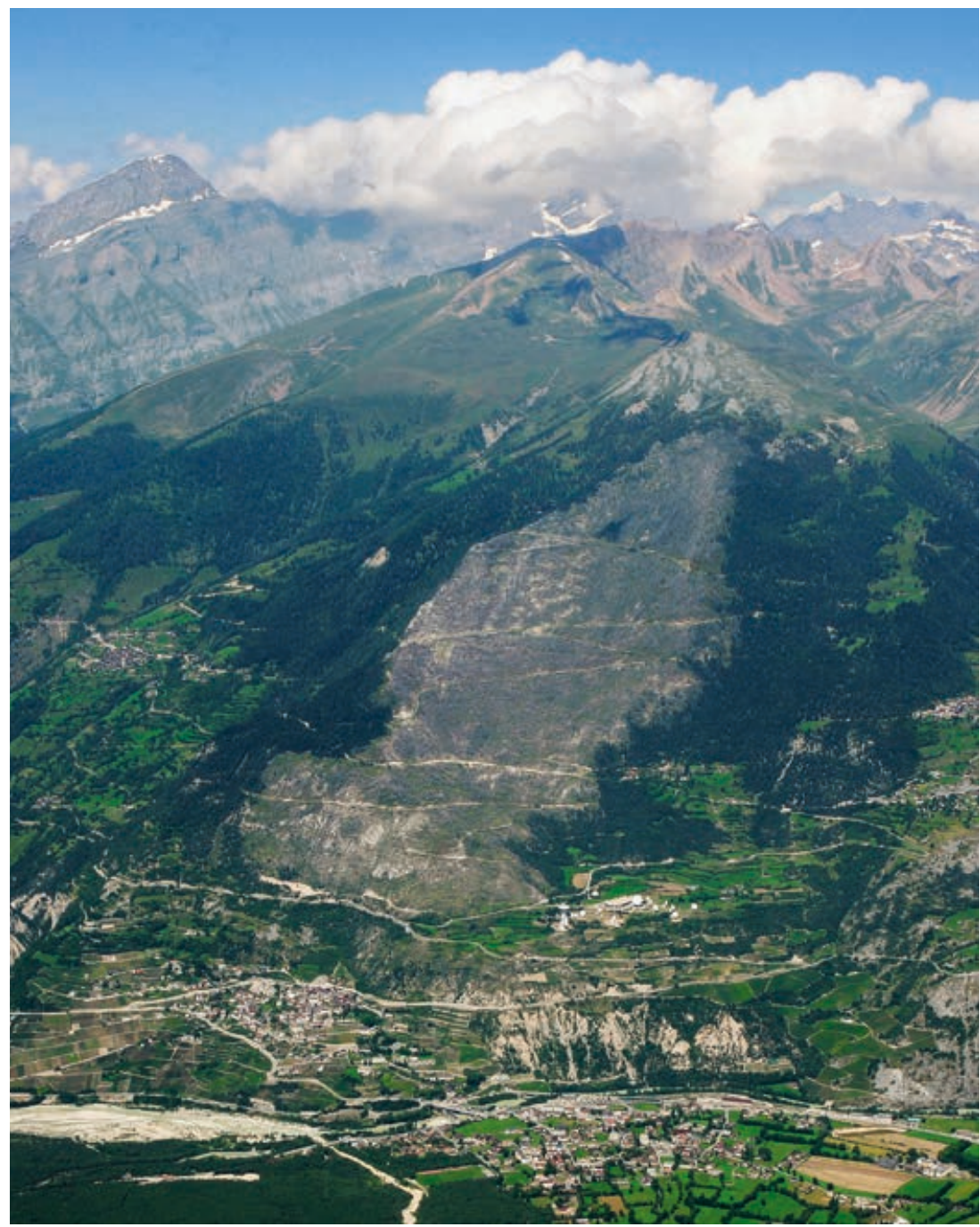

Abb 1 Das Waldbrandgebiet von Leuk im Jahr 2007, vier Jahre nach dem Feuer. Foto: Thomas Wohlgemuth
Waldbrandflächen im Alpenraum sind rar. Im Wallis wurde die Entwicklung der Vegetation nach einem Brand im Gebiet «Bergji-Platten» zwischen Leuk und Erschmatt dokumentiert, wo ein Feuer im Jahr 1979 rund 120 ha Föhrenwald und Felsensteppe verbrannte (Delarze \& Werner 1985, Maumary et al 1995). Nach 13 Jahren wurde eine stark verzögerte Wiederbewaldung im Waldföhrenbestand beobachtet. Für Brandflächen im Tessin haben Delarze et al (1992) eine rasche Wiederbesiedlung nach Waldbrand festgestellt, jedoch eine Verarmung der Flora auf mehrmals abgebrannten Flächen beobachtet. In den Tiroler Kalkalpen wurden anhand von 26 unterschiedlich alten Waldbrandgebieten eine langsam fortschreitende Waldverjüngung in höheren Lagen gefunden und generell ein mehrere Jahrhunderte andauernder Wiederbewaldungsprozess der Brandflächen beobachtet (Sass \& Sarcletti 2017). Im Yellowstone Nationalpark in den USA wuchsen nach einem verheerenden Feuer 1988 jene Baumarten ein, die vor der Störung vorherrschten, hier also Nadelbaumarten (Romme et al 2011). Dieses Phänomen wurde auch auf der Alpensüdseite für die Wiederbesiedlung von Waldbrandflächen in Kastanien- (Delarze et al 1992) und Buchenwäldern (Maringer et al 2016) beschrieben. Da je nach Intensität eines Waldbrandes die herrschende Vegetation teilweise oder vollständig zerstört wird, zudem auch Teile des organischen Bodens sowie die darin enthaltenen Pflanzenwurzeln betroffen sein können (Wohlgemuth et al 2005) und auch die Nährstoffzusammensetzung und -verfügbarkeit stark ändert (Delarze \& Werner 1985), stellt sich unweigerlich die Frage nach möglichen Gesetzmässigkeiten der Sukzession. Mehrere Studien über die Vegetationsentwicklung nach Waldbrand (Capitanio \& Carcaillet 2008, Harvey \& Holzman 2014) berufen sich auf das Konzept der «Artenzusammensetzung der Ausgangsvegetation» von Egler (1954), auf Englisch initial floristic composition, das im Rahmen von nicht mehr bewirtschaftetem Grünland entworfen wurde. Demnach sollen die bereits vorhandenen Arten oder jene Arten, die sich zum Beispiel auf einer gestörten Fläche zuerst etablieren, die späteren Stadien der Vegetationsentwicklung prägen (Wilson et al 1992). Einen anderen Blickwinkel nimmt das Konzept der «Erleichterung der weiteren Besiedlung» ein, auf Englisch facilitation, wonach die Vegetationsentwicklung auf den erstankommenden Arten aufbaut und sich in unterschiedlicher Richtung entwickeln kann (Connell \& Slatyer 1977). Vor dem Hintergrund dieser zwei Konzepte stellen wir folgende Fragen: 1) Wie entwickelte sich die Vegetation in der Waldbrandfläche in den ersten zehn Jahren nach dem Waldbrand? 2) Welche Faktoren erklären die Entwicklung der Vielfalt von Pflanzenarten? 3) Von welchen Faktoren hängt die Besiedlung der Brandfläche mit Baumarten ab? 


\section{Methoden}

Die Brandfläche liegt zwischen zwei Hangrippen und erstreckt sich von Leuk bis zum Oberen Guggerhubel auf $2241 \mathrm{~m}$ ü. M. (Abbildung 1). Vor dem Feuer war dies zusammenhängendes Waldgebiet, das in einem Höhenband von 900 bis $1500 \mathrm{~m}$ ü. M. hauptsächlich aus Waldföhren (Pinus sylvestris) bestand, am unteren Rand stark mit Flaumeichen (Quercus pubescens) durchsetzt war und am oberen Rand in Lärchen-Fichtenwald überging. Bis etwa $1800 \mathrm{~m}$ ü. M. dominierte die Fichte (Picea abies), und oberhalb davon begann die Lärche (Larix decidua) zu herrschen. Im obersten Teil ging der Wald in eine Lärchen-Wytweide über (Werlen 1995). In mittleren

\begin{tabular}{|c|c|c|c|c|c|}
\hline \multirow[t]{2}{*}{ Merkmal } & \multicolumn{5}{|c|}{ Jahre nach dem Brand } \\
\hline & 1 & 2 & 3 & 4 & 10 \\
\hline \multicolumn{6}{|c|}{ Deckungsgrad der Vegetation (\%) } \\
\hline Krautschicht & 9.8 & 35.3 & 66.7 & 73.0 & 73.1 \\
\hline Strauchschicht & 0.4 & 1.2 & 1.6 & 3.3 & 8.9 \\
\hline Baumschicht & 1.5 & 1.5 & 1.8 & 1.6 & 3.6 \\
\hline \multicolumn{6}{|c|}{ Anzahl Pflanzenarten (mittlere Anzahl pro $200 \mathrm{~m}^{2}$ ) } \\
\hline Krautschicht & 28.9 & 42.5 & 46.4 & 47.6 & 45.3 \\
\hline \multicolumn{6}{|c|}{ Frequenz und mittlere Dominanz der in Abbildung 3 dargestellten Arten (\%) } \\
\hline Blitum virgatum & $1(0.1)$ & $37(0.4)$ & $78(7)$ & $71(1.6)$ & $12(0.5)$ \\
\hline Erigeron canadensis & $20(0.3)$ & $78(0.9)$ & $73(2.5)$ & $67(5.1)$ & $35(0.5)$ \\
\hline Calamagrostis varia & $56(2.5)$ & $65(2.9)$ & $76(3.4)$ & $76(4.8)$ & $84(21)$ \\
\hline Epilobium angustifolium & $59(0.5)$ & $85(7)$ & $92(17.7)$ & $92(22.3)$ & $96(6.2)$ \\
\hline \multicolumn{6}{|c|}{ Baumverjüngung (Stämme $\geq 25 \mathrm{~cm}$; Anzahl pro ha) } \\
\hline 900-1299 m ü. M. $(n=53)$ & & & & & \\
\hline Laubbäume & 102 & 487 & 622 & 705 & 1676 \\
\hline Nadelbäume & 84 & 91 & 69 & 95 & 138 \\
\hline 1. Populus tremula & 0 & 164 & 258 & 258 & 874 \\
\hline 2. Salix caprea/S. appendiculata & 0 & 87 & 153 & 164 & 229 \\
\hline 3. Betula pendula & 0 & 18 & 40 & 109 & 246 \\
\hline 4. Pinus sylvestris & 76 & 80 & 69 & 87 & 68 \\
\hline 5. Quercus pubescens & 44 & 80 & 62 & 44 & 117 \\
\hline \multicolumn{6}{|l|}{$1300-1699 m$ m ü $M .(n=60)$} \\
\hline Laubbäume & 38 & 669 & 1117 & 886 & 1925 \\
\hline Nadelbäume & 3 & 3 & 7 & 95 & 93 \\
\hline 1. Populus tremula & 14 & 283 & 476 & 328 & 1073 \\
\hline 2. Salix caprea/S. appendiculata & 21 & 352 & 576 & 431 & 494 \\
\hline 3. Betula pendula & 0 & 21 & 59 & 117 & 303 \\
\hline 4. Larix decidua & 0 & 3 & 7 & 10 & 43 \\
\hline 5. Picea abies & 0 & 0 & 0 & 0 & 45 \\
\hline \multicolumn{6}{|l|}{$1700-2100 m$ ü. M. $(n=37)$} \\
\hline Laubbäume & 31 & 2056 & 2313 & 1749 & 2255 \\
\hline Nadelbäume & 138 & 144 & 174 & 179 & 723 \\
\hline 1. Salix caprea/S. appendiculata & 31 & 1369 & 1528 & 1118 & 1076 \\
\hline 2. Populus tremula & 0 & 651 & 733 & 554 & 1065 \\
\hline 3. Larix decidua & 77 & 72 & 133 & 108 & 350 \\
\hline 4. Picea abies & 62 & 72 & 41 & 72 & 368 \\
\hline 5. Betula pendula & 0 & 21 & 36 & 62 & 97 \\
\hline
\end{tabular}

Tab 1 Eckdaten zur Dynamik der Vegetation nach dem Brand von 2003. Bei der Baumverjüngung sind die fünf häufigsten Arten bezüglich der Summe der Zählungen 2004 bis 2013 rangiert.
Lagen dürften an den östlichen und westlichen Rändern der Brandfläche auch wenige Weisstannen (Abies alba) gestanden haben. Im Rahmen einer Doktorarbeit wurden im Gebiet 40 Waldvegetationsaufnahmen durchgeführt, die einen Vergleich mit der Vegetation vor dem Brand ermöglichen (Gödickemeier 1998, Wohlgemuth et al 2010). Während im untersten Teil noch Spuren von früheren Ackerterrassen vorhanden sind, zeugen Steinmauern im mittleren Teil des Brandgebiets von früherer Beweidung. Spuren von jüngeren Waldeingriffen fehlen dagegen weitgehend. Die Burgergemeinde Leuk besitzt 90\% des vom Brand betroffenen Waldgebietes. Ihr Vorschlag, einen Grossteil aufzuforsten, wurde vom Kanton nicht unterstützt, weshalb sie auf waldbauliche Eingriffe weitgehend verzichtete. An vier Stellen wurden kleinflächige Testpflanzungen mit verschiedenen Laubbaumarten durchgeführt.

Innerhalb des Perimeters der Brandfläche wurden 2004153 quadratische Stichprobenflächen (SPF) von je $200 \mathrm{~m}^{2}$ effektiver Grösse festgelegt (mit zunehmender Hangneigung nimmt die Projektionsfläche der SPF ab). Die Stichprobenzentren liegen jeweils $125 \mathrm{~m}$ voneinander entfernt, sind am Koordinatensystem der Schweizer Landeskarte ausgerichtet und mit Metall-T-Profilen dauerhaft markiert (Wohlgemuth et al 2005). In den Jahren 2004, 2005, 2006, 2007 und 2013, also 1, 2, 3, 4 und 10 Jahre nach dem Brand, wurden jeweils in den Monaten Juli und August auf den SPF Daten über die Vegetation erhoben. Von allen Farn- und Blütenpflanzen sowie den häufigsten Moosarten wurden die Flächenanteile mit der verfeinerten Londo-Skala (Londo 1975) in Prozentklassen geschätzt. Auf einem Viertel der SPF (Dreieck mit $50 \mathrm{~m}^{2}$ Grösse zwischen Flächenzentrum und den beiden östlichen Eckpunkten) wurde die Verjüngung der Baumarten in vier Höhenklassen (1-24 cm, inklusive Sämlinge; 25-99 cm; $100-199 \mathrm{~cm} ; \geq 200 \mathrm{~cm}$ ) gezählt. 2013 wurden die Individuen aller Baumarten sowohl auf der Teilfläche von $50 \mathrm{~m}^{2}$ als auch auf Gesamtfläche von $200 \mathrm{~m}^{2}$ gezählt. Im Verlaufe der Jahre sind fünf Flächen durch Bauten zerstört worden oder wurden nicht mehr gefunden. Für die vorliegende Auswertung wurden 148 SPF berücksichtigt.

Trendlinien für Artenzahlen entlang des Höhengradienten wurden mittels generalisierter additiver Modelle (GAM) berechnet. Mit generalisierten linearen Modellen (GLM) wurden Zusammenhänge zwischen Artenzahlen und verschiedenen Einflussfaktoren festgestellt. Hierzu dienten folgende Faktoren als Grundlage: Höhe ü. M. als Proxi-Variable, entlang welcher die mittlere Jahrestemperatur abnimmt und die Niederschlagssummen zunehmen, die Projektionsfläche $\left(\mathrm{m}^{2}\right.$; abhängig von der Neigung der SPF), die Distanz zum intakten Wald und zur nächsten Wiese/Weide sowie die im Jahr 2004 gemessenen Faktoren unbedeckte Bodenoberfläche (\% der 


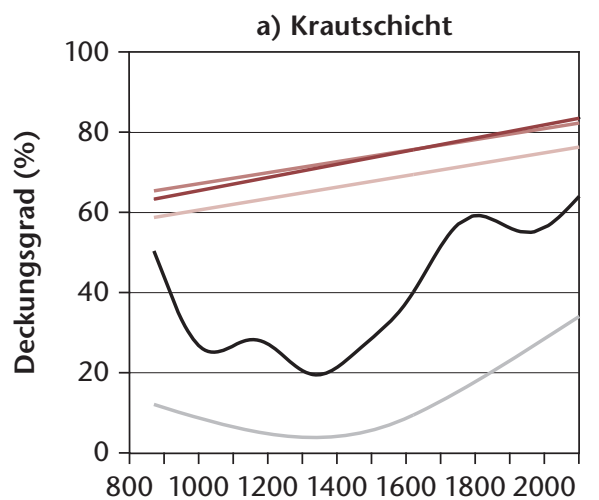

Höhe (m ü. M.) b) Strauchschicht

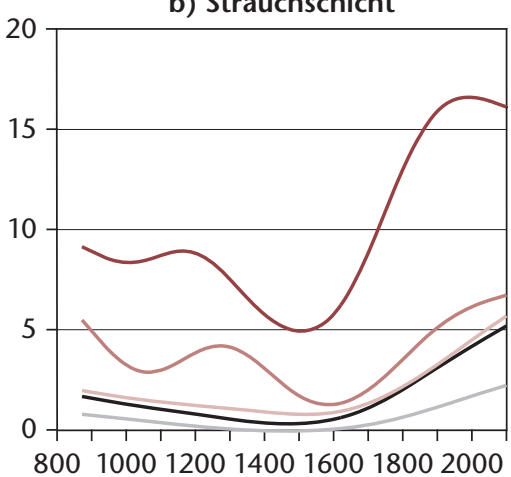

Höhe (m. ü. M.)
Zeit nach dem Brand

$$
\text { - } 10 \text { Jahre }-4 \text { Jahre }-3 \text { Jahre }-2 \text { Jahre - } 1 \text { Jahr }
$$

Abb 2 Entwicklung des Deckungsgrads der Krautschicht (a) und der Strauchschicht (verholzte Pflanzen 0.5-5.0 m; b) mit zunehmender Zeit nach dem Brand von 2003. Der Zusammenhang zwischen den Deckungsprozenten und der Meereshöhe wurde mit GAMModellen berechnet.

\section{a) Erdbeerspinat (Blitum virgatum)}

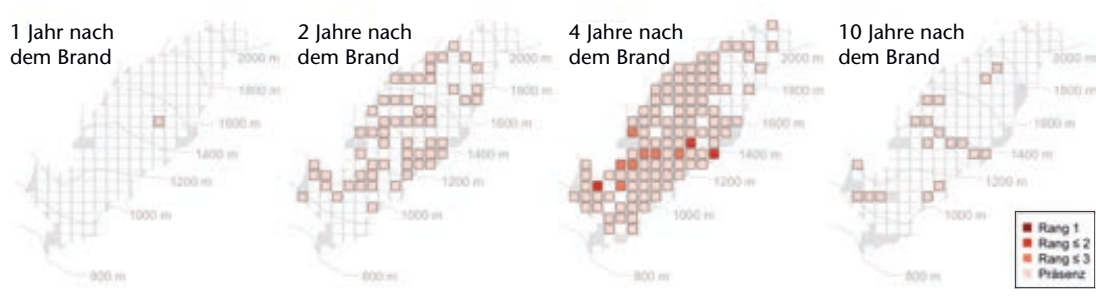

b) Kanadisches Berufskraut (Conyza canadensis)
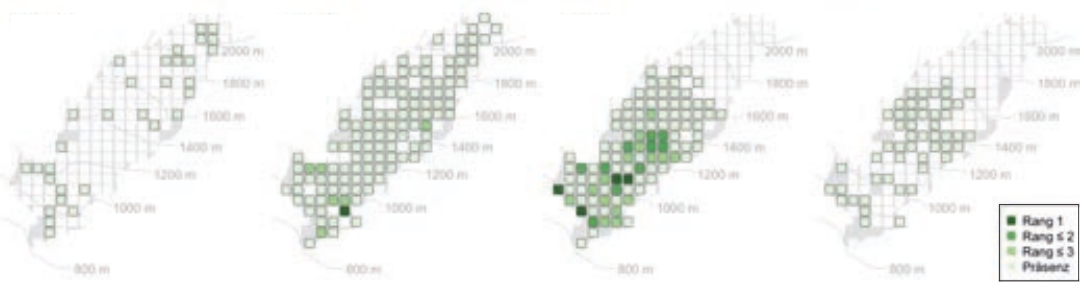

c) Wald-Weidenröschen (Epilobium angustifolium)


d) Buntes Reitgras (Calamagrostis varia)
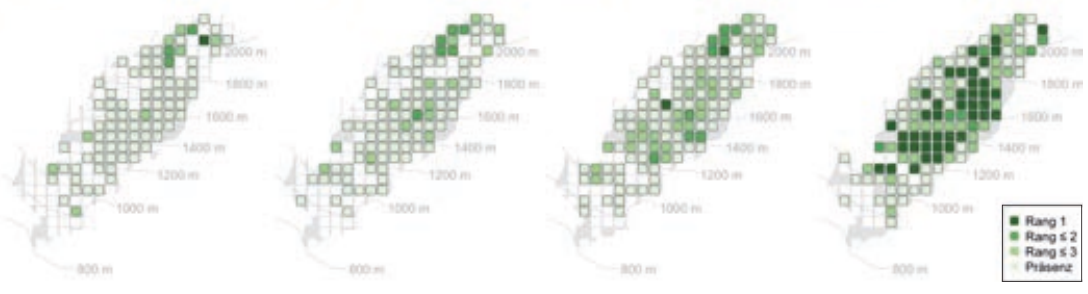

Abb 3 Muster der Wiederbesiedlung von vier Pflanzenarten nach dem Brand von 2003 basierend auf Vegetationsaufnahmen auf den 200- $m^{2}$-Flächen. Die Farben entsprechen dem Erscheinungsbild der Art zur Blütezeit, die Stärke der Farben gibt ihre Dominanz bezüglich aller anderen Pflanzenarten in der Vegetationsaufnahme an: Rang 1 entspricht der Art in der Stichprobenfläche (SPF) mit der grössten Flächenbedeckung. Die SPF sind überproportional gross dargestellt.
Gesamtfläche), Anteil Fels/Stein (\% der Gesamtfläche), Mächtigkeit der Ascheauflage (cm; gemittelt aus 9 Bohrstock-Messungen pro SPF; Indikator für die Brandintensität) und die Bodentiefe (cm; gemittelt aus 8 Bohrstockmessungen). Für die Datenauswertung und für die Darstellung der Resultate wurde das Statistikprogramm R verwendet (R Development Core Team 2016).

\section{Resultate}

\section{Vegetationsdecke}

Die Waldbrandfläche war ein Jahr nach dem Feuer im Durchschnitt zu 9.8\% mit Vegetation bedeckt, wobei ein Viertel der SPF weniger als 1\% Vegetationsbedeckung aufwies (Tabelle 1, Abbildung 2a). Während zwei Jahre nach dem Feuer noch die Hälfte aller SPF 30\% und weniger Vegetation aufwiesen, verdichtete sich die Pflanzendecke im dritten Jahr bereits auf 60 bis 75\% auf allen SPF, ein Niveau, das in den nächsten Jahren nur noch wenig überschritten wurde. In mittleren Höhenlagen (1000-1600 m ü. M.), wo die horizontale Flächenausdehnung am grössten war und das Feuer besonders stark brannte, verlief die Wiederbesiedlung langsamer.

\section{Besiedlung durch Baumarten}

Die Dynamik der Wiederbesiedlung mit Baumarten lässt sich während der ersten zehn Jahre anhand ihres Deckungsgrades verfolgen (alle Baumarten bis $5 \mathrm{~m}$ Höhe; der Strauchschicht zugehörig; Abbildung 2b). Während die Waldföhre, die Fichte, die Lärche, die Aspe (Populus tremula), die Birke (Betula pendula) und die Weiden (Salix caprea/S. appendiculata) die Fläche über Samenflug und -keimung langsam wiederbesiedelten, vermehrte sich die Flaumeiche zu Beginn vorwiegend über Stockausschlag aus den offensichtlich intakten Wurzeln von oberflächlich abgebrannten Individuen. Während mit zunehmender Zeit nach dem Feuer die Eiche über den Eichelhäher (Garrulus glandarius) ausgebreitet wurde, breitete sich die Pappel nach erfolgreicher Erstbesiedlung unterirdisch weiter aus und bildete oft mehrere Meter entfernt vom ersten Stamm neue Triebe. Zehn Jahre nach dem Feuer bedeckten Bäume und Sträucher unterhalb von $1800 \mathrm{~m}$ ü. M. maximal 10\% der SPF, weiter oben betrug deren Deckungsgrad im Mittel über 15\%.

\section{Besiedlung durch Kräuter und Süssgrasartige}

Während der ersten zehn Jahre sind 560 Pflanzenarten in den SPF gefunden worden. Eine besonders eindrückliche Dynamik der Wiederbesiedlung war zwei bis vier Jahre nach dem Feuer festzustellen, als viele Samen durch Wind oder Tiere in das Brandgebiet gelangten und gleichzeitig viele Nährstoffe durch Kationenfreisetzung aus der Asche verfügbar 
a) Alle Arten



d) Stauden, Zwergsträucher

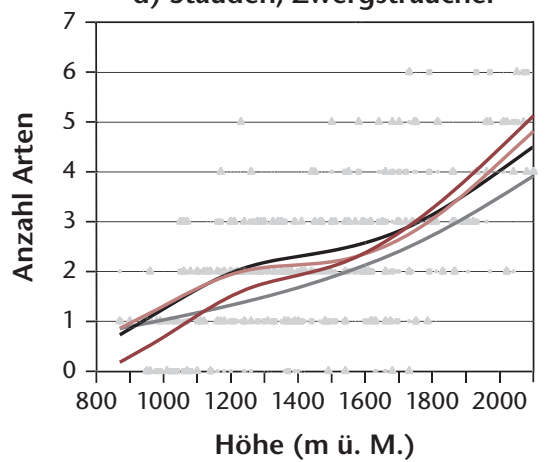

b) Kräuter

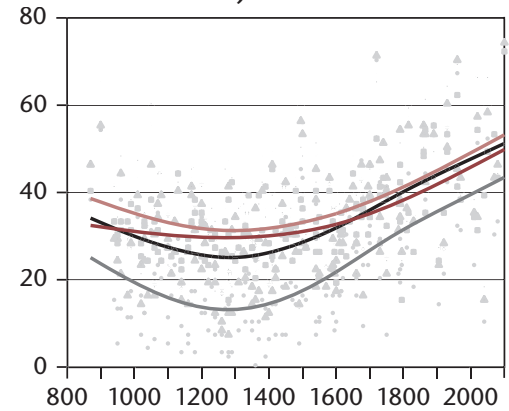

e) Sträucher



c) Süssgrasartige

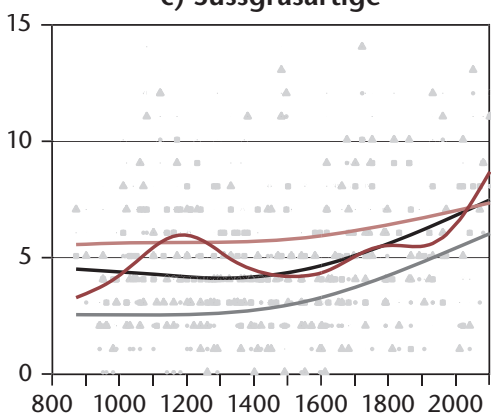

f) Bäume

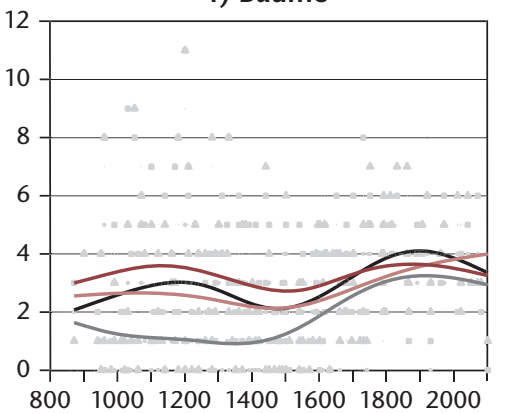

Höhe ( $m$ ü. M.)

Zeit nach dem Brand

- 10 Jahre $\quad 4$ Jahre - 2 Jahre -1 Jahr

Abb 4 Veränderung der Artenvielfalt im Waldbrandgebiet entlang der Höhe über Meer für alle Arten sowie für ausgewählte Artengruppen. In der Artengruppe «Süssgrasartige» sind Gräser, Seggen, Binsen und Simsen zusammengefasst. Der Zusammenhang zwischen Artenvielfalt und Meereshöhe wurde mit der GAM-Methode berechnet.

wurden (hierzu weitere Resultate in Wohlgemuth et al 2005, Wohlgemuth \& Moser 2009, Wohlgemuth et al 2010). Anhand von vier ausgesuchten Arten kann die Dominanzveränderung in der Brandfläche exemplarisch aufgezeigt werden (Tabelle 1, Abbildung 3). Der Erdbeerspinat (Blitum virgatum; Abbildung 3a), eine Pionierart auf Offenflächen, war ein Jahr nach dem Feuer in nur einer SPF gefunden worden. Zwei Jahre später war die im Wallis nur vereinzelt vorkommende Art in mehr als drei Vierteln aller SPF vorhanden und in drei Flächen gar die dominante Art mit bis zu 70\% Bodenbedeckung. Nach dieser maximalen Abundanz nahmen sowohl Frequenz als auch Häufigkeit stark ab. Ähnlich pulsartig erschien das Kanadische Berufskraut (Conyza canadensis; Abbildung 3b), der in Europa häufigste Neophyt. Im ersten Jahr nach dem Feuer war es in $20 \%$ der SPF präsent, im zweiten Jahr bereits in $80 \%$. Gleich wie beim Erdbeerspinat waren die Deckungsprozente im dritten Jahr nach dem Feuer am grössten. Am häufigsten war das Berufskraut in den tiefer gelegenen Flächen, während die Höhenbegrenzung auf ca. $1600 \mathrm{~m}$ ü. M. im vierten Jahr nach dem Feuer auf eine Temperaturlimitierung hinweist. Im Gegensatz zu diesen Pionierpflanzen breiteten sich andere Arten während der ersten zehn Jahre nach dem Feuer kontinuierlich aus. Zwei der häufigsten Arten waren das Wald-Weidenröschen (Epilobium angustifolium; Abbildung 3c) und das Bunte Reitgras (Calamagrostis varia; Abbildung 3d). Das Weidenröschen dehnte in den ersten zwei Jahren sein Areal von den höheren in die tieferen Lagen aus. Bereits im zweiten Jahr wuchs es in $85 \%$ aller SPF, und im vierten Jahr nach dem Feuer dominierte es in einem Drittel derselben. Auch nach zehn Jahren rangierte es unter den häufigsten Arten. Ähnlich weit verbreitet war auch das Bunte Reitgras, doch erreichte das Gras seine vorderhand grösste Ausdehnung erst nach rund zehn Jahren.

\section{Artenzahlen}

Die Artenvielfalt entwickelte sich während der ersten drei Jahre nach dem Feuer rasant. Trotz geringer Bedeckung mit Pflanzen waren im ersten Jahr auf den SPF im Durchschnitt bereits 28.9 Arten zu finden (Tabelle 1, Abbildung 4a). Diese Zahl erhöhte sich bis ins vierte Jahr nach dem Feuer auf durchschnittlich 47.6 Arten und betrug nach zehn Jahren immer noch 45.3 Arten pro SPF. Höchste Artenzahlen wurden in den obersten Höhenlagen erreicht und beliefen sich im Übergang vom Lärchen-Fichtenwald zur Lärchenweide auf über 100 Arten pro $200 \mathrm{~m}^{2}$. Die Muster sind stark mit der Artengruppe der Kräuter (Abbildung 4b) korreliert. Gräser, Seggen, Binsen und Simsen, die nach heutiger Systematik zu den Süssgrasartigen zusammengefasst sind (Baltisberger et al 2013), waren nach zehn Jahren in Lagen um $1200 \mathrm{~m}$ ü. M. und nahe der Waldgrenze am zahlreichsten (Abbildung 4c). Stauden und Zwergsträucher der Gattungen Vaccinium, Arctostaphylos, Poly- 


\begin{tabular}{|c|c|c|c|c|c|c|c|c|c|c|c|c|c|c|c|}
\hline & \multirow{2}{*}{\multicolumn{4}{|c|}{1}} & & \multirow{2}{*}{\multicolumn{4}{|c|}{10}} \\
\hline & & & & & & 2 & & & 4 & & & & & & \\
\hline Erklärte Varianz des Modells ( $\mathrm{D}^{2}$ ) & \multicolumn{4}{|c|}{$63 \%$} & \multicolumn{3}{|c|}{$52 \%$} & \multicolumn{4}{|c|}{$53 \%$} & \multicolumn{4}{|c|}{$51 \%$} \\
\hline Achsenabschnitt & \multicolumn{4}{|c|}{$4.42^{* * *}$} & \multicolumn{3}{|c|}{$4.39 * * *$} & \multicolumn{4}{|c|}{$4.18^{* \star *}$} & \multicolumn{4}{|c|}{$3.56^{* * *}$} \\
\hline Höhe ü. M. (m) & -0.82 & ** & 1.53 * & *** & -0.74 & $* * *$ & $1.2 * * *$ & -0.94 * & $* * *$ & 1.25 * & *** & -1.14 & $* * *$ & 1.55 & *** \\
\hline Unbedeckte Bodenoberfläche (2004; \%) & $2.13 *$ & $* * *$ & $-2.09 *$ & *** & 1.31 & $* * *$ & $-1.34 * * *$ & 1.45 & $* * *$ & $-1.51 *$ & *** & 0.47 & • & -0.44 & \\
\hline Projektionsfläche $\left(\mathrm{m}^{2}\right)$ & $-1.19 *$ & $* * *$ & $1.39 *$ & *** & -0.63 & ** & $0.76 * * *$ & 0.07 & & 0.17 & & 0.68 & ** & -0.48 & * \\
\hline Bodentiefe $(2004 ; \mathrm{cm})$ & -0.76 & * & 0.21 & & -0.28 & & 0.09 & 0.13 & & -0.2 & & 0.76 & ** & -0.7 & ** \\
\hline Distanz zum intakten Wald $(\mathrm{m})$ & -0.30 & & 0.52 & * & 0.19 & & 0 & 0.18 & & -0.06 & & -0.06 & & 0.12 & \\
\hline Distanz zur nächsten Wiese/Weide (m) & -0.13 & & -0.17 & & -0.42 & * & 0.27 & -0.5 & * & 0.35 & • & 0.1 & & -0.06 & \\
\hline
\end{tabular}

Tab 2 Resultate der Regressionsanalysen (General Linear Models, GLM). Angegeben sind die Schätzer der standardisierten Variablen mit linearem und quadratischem Term, womit deren Grösse direkt dem Einfluss auf die Artenzahl entspricht. Folgende Signifikanzniveaus sind unterschieden: ${ }^{* *}<0.0001$, ${ }^{* *}<0.001,{ }^{*}<0.01, \cdot<0.05$.

gala und Rubus (Brombeeren und Himbeeren) waren in tiefen Lagen wenig zahlreich und nahmen gegen die Waldgrenze kontinuierlich zu (Abbildung 4d). Die mittlere Anzahl Baumarten in der Strauchschicht hat in Lagen unterhalb von $1700 \mathrm{~m}$ ü. M. vom ersten zum zweiten Jahr nach dem Feuer deutlich zugenommen, ist in den nachkommenden Jahren aber erstaunlich konstant geblieben (Abbildung 4f). Bis auf etwa $1200 \mathrm{~m}$ ü. M. wurden pro SPF im Durchschnitt drei bis vier Straucharten gefunden, darüber noch ein bis zwei Arten (Abbildung 4e). Im Gegensatz dazu wurden auf allen Höhenstufen drei bis vier Baumarten pro SPF angetroffen.

Die Resultate aus den Regressionsanalysen zeigen, dass die Anzahl Pflanzenarten im Brandgebiet in allen untersuchten Jahren stark mit der Höhe über Meer zusammenhing, wobei die Werte in den ersten Jahren nach dem Feuer im unteren und oberen
Teil des Gebiets deutlich grösser waren als in mittleren Lagen (Tabelle 2). Im mittleren Teil wurde im ersten Jahr am wenigsten Vegetation festgestellt, gleichbedeutend mit grossen Anteilen an unbedeckter Bodenoberfläche und Fels/Stein. Für die Artenvielfalt war die unbedeckte Bodenoberfläche ein ebenso wichtiger Einflussfaktor wie die Meereshöhe. Nach zehn Jahren rangierten Bodentiefe und Projektionsfläche neben der Meereshöhe als signifikante Prädiktoren der Artenvielfalt. Dagegen nahm die Bedeutung der unbedeckten Bodenoberfläche und von Fels/Stein ab. Eine hohe Vielfalt herrschte demnach gegen die Waldgrenze und an Orten mit geringer Bodentiefe, an denen Arten noch auf unbedecktem Boden keimen konnten.

Überall in der Brandfläche haben sich Bäume etabliert. Die Dichten zehn Jahre nach dem Feuer betrugen zwischen 1800 und 3000 Stämmen $\geq 25 \mathrm{~cm}$
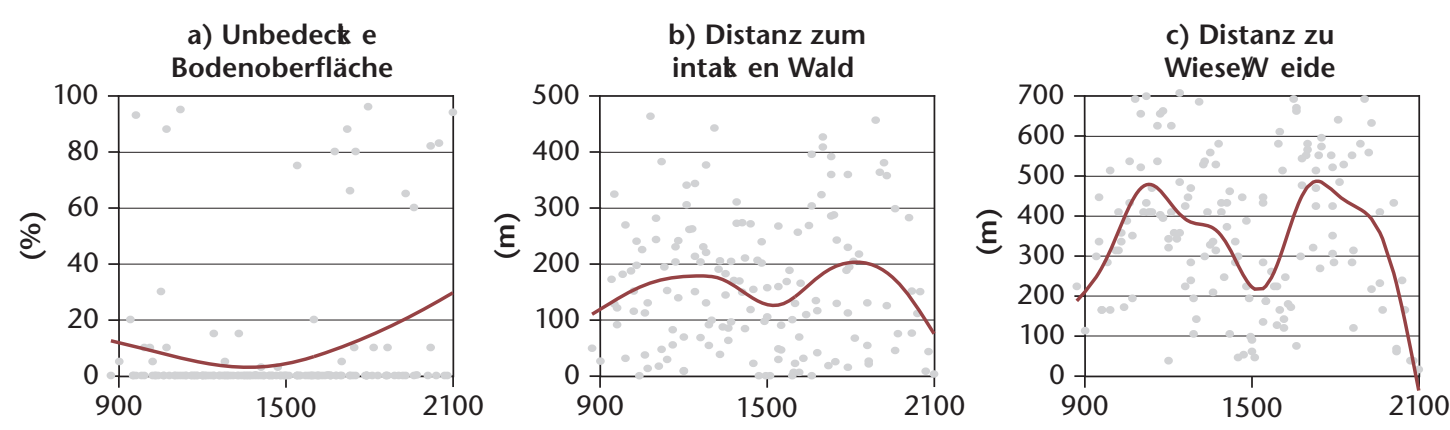

d) Aschemächtigkit


Abb 5 Zusammenhang zwischen der Meereshöhe und den untersuchten Variablen, dargestellt mit GAM-Kurvenfittern. 


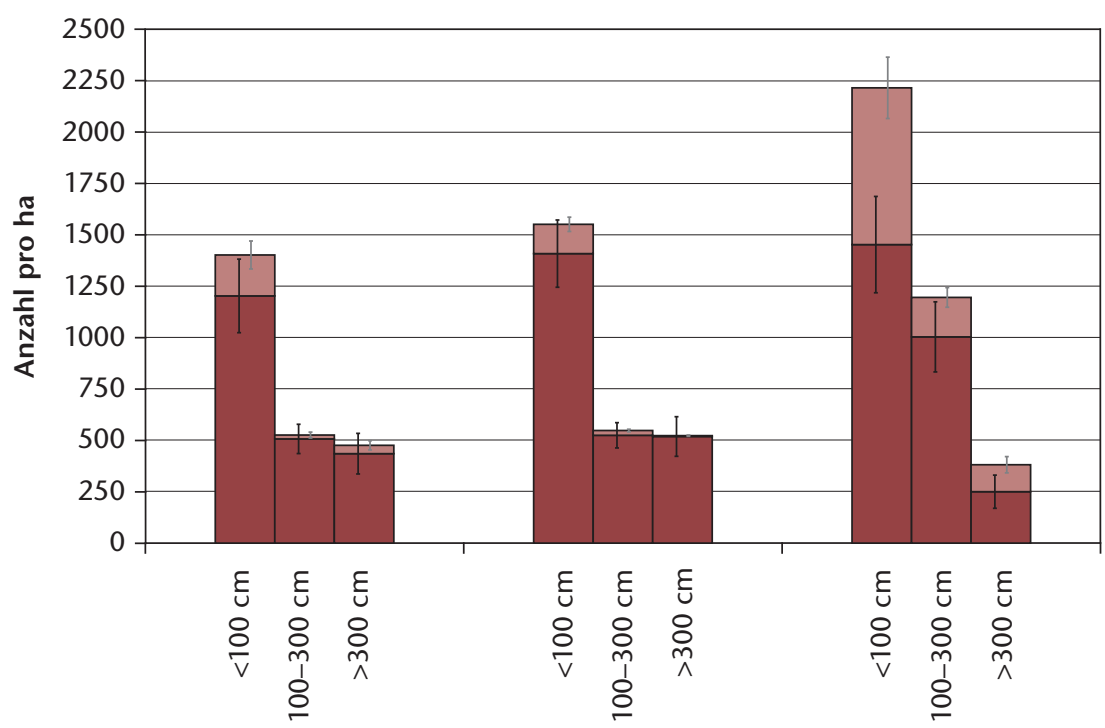

900-1299 m ü. M. $\quad$ 1300-1699 m ü. M. $\quad$ 1700-2100 $m$ ü. M.

- Laubbäume $\quad$ Nadelbäume

Abb 6 Verjüngung auf der Brandfläche zehn Jahre nach dem Feuer, gruppiert in drei Höhenstufen, drei Grössenklassen und unterteilt in Nadel- und Laubbäume. Fehlerbalken geben die Variation in den drei Höhenstufen an. Grundlage für die Berechnung waren die Zählungen auf der gesamten Stichprobenfläche $\left(200 \mathrm{~m}^{2}\right)$.

pro Hektare (Tabelle 1, Abbildung 6). Der unterste Teil der Brandfläche (900-1299 m ü. M.) war mit mittleren 1800 Stämmen pro Hektare dünn bis lückig und hauptsächlich mit Laubbäumen $(92 \%$ der Verjüngung) bestockt, insbesondere mit Aspen, Weiden, Birken und Flaumeichen. Als fünfthäufigste Baumart verjüngte sich die Waldföhre, und zwar besonders in der Nähe von Samenbäumen. In der mittleren Höhenstufe (1300-1699 m ü. M.) machten Laubbäume gar 95\% der 2000 Stämme pro Hektare aus. Ab $1700 \mathrm{~m}$ ü. M. bis zur Waldgrenze etablierten sich vermehrt Nadelbäume (ca. 25\% der Verjüngung) unter den rund 3000 Stämmen pro Hektare, wobei Fichte und Lärche etwa gleich häufig (je 12\%) anzutreffen waren. Zehn Jahre nach dem Feuer dominierte die Aspe in allen Höhenlagen mit Dichten von 870 bis 1070 Stämmen pro Hektare. Die Weiden waren im obersten Teil gleich häufig und weiter unten etwa halb so häufig wie die Aspe. Als dritthäufigste Art in der gesamten Fläche rangierte die Birke.

\section{Diskussion}

Waldbrände sind aus Sicht des Menschen verheerende Ereignisse, die je nach Dimension grosse Schäden anrichten und eine langfristige Veränderung in der Landschaft bewirken. Störungen und die darauf folgenden Prozesse der natürlichen Wiederbesiedlung geben aber wichtige Anhaltspunkte zur Regenerationsfähigkeit der Natur. Dies ist heute im Zeitalter des Klimawandels umso bedeutsamer, weil höhere Temperaturen und ausgeprägte Trockenperioden alle Ökosysteme beeinflussen (Allen et al 2015) und weil die Reaktionen der einzelnen Bäume und ganzer Wälder auf die Umweltveränderungen nur schwer abschätzbar sind. Hierbei spielt auch die Dimension der Störungen eine Rolle. Auf kleinflächige Störungen reagieren Wälder rasch durch Zuwachsen der Kronen, direkten Einwuchs durch Samen von unbeschädigten Bäumen oder durch Aufwuchs junger unbeschädigter Jungpflanzen (z.B. Kramer et al 2014, Maringer et al 2016). Entwicklungen auf grösseren Flächen sind dagegen generell heterogener bezüglich der Artenzusammensetzung und der Besiedlungsdynamik (White \& Jentsch 2001). In der weltweit umfassendsten Studie zur Feuerökologie im Yellowstone Nationalpark in den USA, wo mehrere Feuer im Jahr 1988 rund 570000 ha Wald verbrannten, wurde diese grosse Heterogenität der Besiedlungsprozesse bestätigt, ja sogar als Regel deklariert (z.B. Romme et al 2011, Donato et al 2016, Harvey et al 2016). Auch wenn die Waldbrände in der Schweiz um mehrere Grössenordnungen kleiner sind, lassen sich gleichwohl spannende Schlüsse aus einer Langzeitbeobachtung ableiten. So zeigt unsere Arbeit, dass selbst in der trockensten Region der Schweiz nach einer starken Veränderung der Bodenund Lichtverhältnisse innerhalb von wenigen Jahren wieder eine artenreiche Pflanzendecke entsteht, die im Vergleich zur ursprünglichen Waldvegetation um 20 bis $40 \%$ mehr Pflanzenarten auf $200 \mathrm{~m}^{2}$ aufweist (Wohlgemuth et al 2010). Die vorübergehend grosse Blütenpracht ist Grundlage für eine reichhaltige Fauna (Moretti et al 2018, dieses Heft).

\section{Vegetationsentwicklung}

Innerhalb von nur drei bis vier Jahren entwickelte sich über der kahlen Oberfläche eine durchschnittlich 60 bis $80 \%$ deckende Vegetationsschicht - ein Wert, der auch nach weiteren sechs Jahren nicht überschritten wurde. Mit zunehmender Zeit nach dem Feuer bildeten die sich ansiedelnden Baumarten ein immer dichteres Gebüsch. Die mittlere Bodenüberdeckung der Bäume variierte nach zehn Jahren zwischen 5 und 20\%. Am geringsten war der Baumbewuchs in einer Zone zwischen 1300 und $1700 \mathrm{~m}$ ü.M., wo wegen des flachgründigen Bodens bereits vor dem Feuer in einem beträchtlichen Teil der Brandfläche nur ein lockerer Wald stand. Generell verlangsamten unterhalb von $1300 \mathrm{~m}$ ü. M. verschiedene Faktoren die Wiederbewaldung. Eine Vegetationserhebung 13 Jahre nach dem Feuer im etwas tiefer gelegenen Brandgebiet «Bergji-Platten» (750-900 m ü. M.) zeigte, dass die vormals mit Föhren bestockte Fläche von Gräsern überwachsen worden war, wodurch die Etablierung von jungen Föhren praktisch verunmöglicht wurde (Maumary et al 1995). Auch in unserer zehnjährigen Untersuchung begannen, ähnlich wie in Windwurfflächen (Wohlgemuth \& Kramer 2015), nach wenigen Jahren Stauden, Süssgrasartige oder 

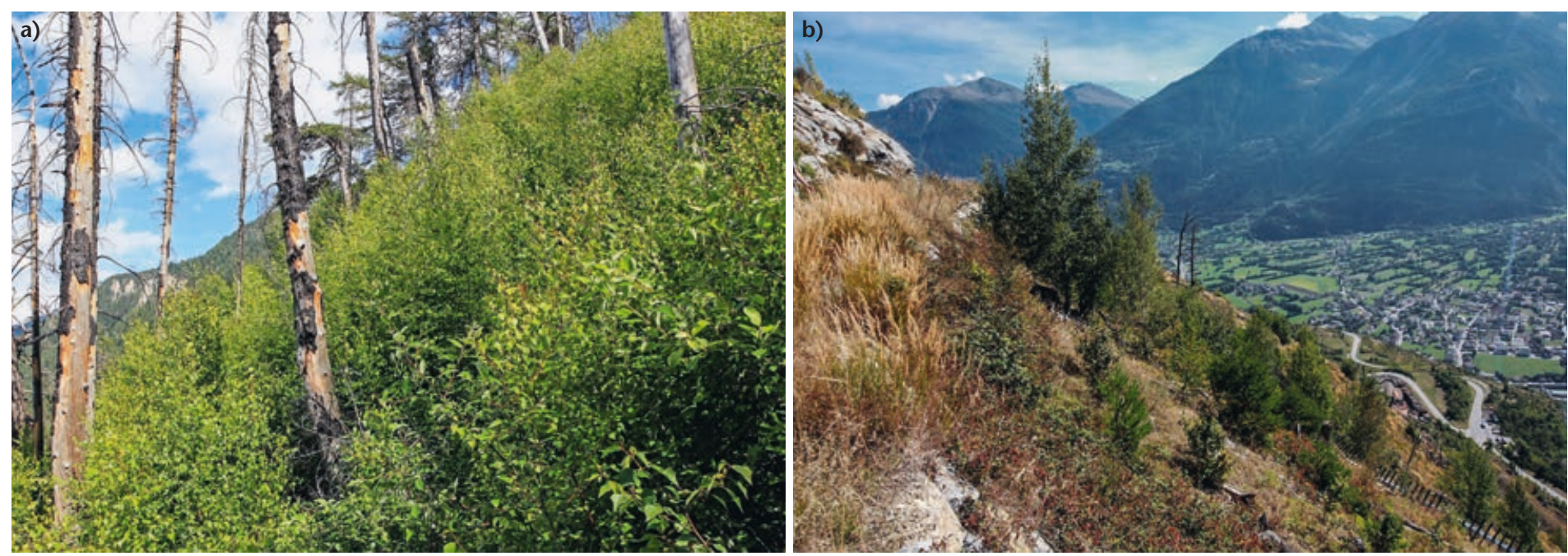

Abb 7 a) Pionierbaumarten prägen die Wiederbesiedlung im mittleren und oberen Teil der Brandfläche (Aufnahme vom Juli 2016). b) Im unteren Teil der Brandfläche schreitet die Verjüngung langsam und unter Beteiligung mehrerer Baumarten voran (Aufnahme vom September 2016). Fotos: a) Ulrich Wasem,

b) Thomas Wohlgemuth

Kräuter zu dominieren. Auffällig dicht wachsen Brombeeren (Rubus caesius) im untersten Teil der Fläche, Reitgräser im mittleren (Calamagrostis varia) und höchsten Teil (C. villosa) sowie Wald-Weidenröschen (Epilobium angustifolium) oberhalb von ca. 1200 m ü. M. (Wohlgemuth \& Moser 2009).

\section{Artenvielfalt}

Die Vielfalt an Pflanzenarten erreichte auf den $200 \mathrm{~m}^{2}$ grossen Aufnahmeflächen Werte, die im dritten und vierten Jahr nach dem Feuer um rund 20 bis $40 \%$ höher waren als im benachbarten, unverbrannten Wald (Wohlgemuth et al 2010). Diesen Vergleich verdanken wir einer früheren Vegetationsstudie im Gebiet (Gödickemeier 1998) sowie mehreren Bachelor- und Masterarbeiten, mithilfe derer wir auf 40 Referenzflächen die Vegetationszusammensetzung vor und nach dem Brand direkt vergleichen konnten (Küttel 2004, Serena 2005, Temperli 2007). Während in Studien zur Sukzession nach Waldbrand im Mittelmeergebiet (Capitanio \& Carcaillet 2008) und in Kalifornien (Harvey \& Holzman 2014) die Pflanzenvielfalt zwei Jahre nach dem Feuer am grössten war, wurden im Brandgebiet von Leuk Höchstwerte im dritten bis vierten Aufnahmejahr nach dem Feuer festgestellt. Diese wurden bei der nächstfolgenden Erhebung, die zehn Jahre nach dem Feuer stattfand, nicht mehr erreicht. Auf mehreren Flächen schritt die Wiederbesiedlung der verbrannten Oberfläche nur langsam voran.

So stiegen die Artenzahlen an Orten, wo sowohl der Anteil der unbedeckten Bodenoberfläche als auch die Ascheauflage am grössten war, nur allmählich an. An diesen Orten war die Intensität des Feuers sehr stark, wodurch zum einen die Samenkeimung von Pflanzen auf der anfangs wasserundurchlässigen Ascheschicht stark eingeschränkt war (Fonturbél et al 2011). Zum andern wurden an Orten von intensivem Feuer nicht nur ein grosser Teil der Humusauflage, sondern auch die im Boden überdauernden Samen vernichtet, was für mehrere Jahre eine geringere Artenvielfalt zu Folge hatte. Ein negativer Zusammenhang zwischen der Brandintensität und der Pflanzenartenvielfalt wurde auch im Yellowstone Nationalpark (Turner et al 1997) und im kalifornischen Buschland (Keeley et al 2008) festgestellt. Der Einfluss der Feuerintensität auf die Artenvielfalt war in Leuk selbst im vierten Jahr nach dem Feuer noch feststellbar. Erstaunlicherweise spielten die Distanzen zum intakten Wald und zu umliegenden Wiesen und Weiden keine entscheidende Rolle für die Pflanzenvielfalt. Entweder breiteten sich die Waldund Grünlandarten nach dem Feuer über weite Strecken gleichmässig rasch aus, oder die Samenbank im Boden mit vornehmlich pionierartigen Pflanzen fiel insgesamt stärker ins Gewicht als die Artengruppe der Grünlandarten. Hierfür spricht die Ausbreitung des Erdbeerspinats, der sich zwei bis drei Jahre nach dem Feuer vornehmlich aus überdauernden Samen etablierte (Moser et al 2006). Die Vielfalt der Pflanzenarten erreichte in den höheren Lagen rund 20 bis 30 Arten mehr als in mittleren und tiefen Lagen. Dieser Anstieg der Vielfalt hängt stark mit den Gebirgsarten zusammen, die an der Waldgrenze und darüber in den Alpweiden vorkommen und in den unteren Lagen vollständig fehlen.

\section{Waldverjüngung}

Für die Besiedlung von Baumarten in Windwurf- und Waldbrandgebieten spielen die bereits vorhandene Verjüngung, die Samenausbreitung und die Keim- und Wuchsbedingungen eine wichtige Rolle (White \& Jentsch 2001). Im Gegensatz zu Windwurfflächen (Kramer et al 2014, Wohlgemuth \& Kramer 2015) zerstört intensives Feuer wie in der Leuker Brandfläche die Verjüngung unter Schirm beinahe 
vollständig. Die Aspe breitete sich nach dem Brand zuerst über Samen flächendeckend aus und vergrösserte ihre Stammzahl während der folgenden Jahre durch Wurzelbrut deutlich. Die Stammdichte auf der gesamten Brandfläche (im Mittel 2180 pro Hektar) liegt im Vergleich mit jener auf Windwurfflächen von Vivian und Lothar im unteren Bereich, ist für zentralalpine Wälder aber nicht aussergewöhnlich (Wohlgemuth et al 2017).

Bereits in einer früheren Analyse der Baumverjüngung in Leuk wurde erkannt, dass die Dichte der Schlussbaumarten Waldföhre, Fichte und Lärche mit zunehmender Distanz zum intakten Wald abnimmt (Moser et al 2010; Wohlgemuth et al 2018, dieses Heft), ein Resultat, das auch in Waldbrandflächen im Aostatal gefunden wurde (Vacchiano et al 2014). Während im oberen Teil der Brandfläche eine rasche und gleichzeitige Wiederbesiedlung durch Weiden, Aspen, Fichten und Lärchen beobachtet wurde (Abbildung 7a), führten in tieferen Lagen mehrere Prozesse zu einem oft nur sehr geringen Bewuchs mit Baumarten (Abbildung 7b). So sind zum Beispiel viele der in den ersten Jahren angeflogenen Waldföhrensamen infolge der heiss-trockenen Sommerwitterung von 2004 und 2005 nach der Keimung vertrocknet (Moser et al 2010). Während sich hier also nur wenige Waldföhren, insbesondere in der Nähe der Mutterbäume, zu etablieren begannen, trieben die wenigen verkohlten Flaumeichen im untersten Teil wieder aus, womit ein Baumartenwechsel einsetzen könnte (Wohlgemuth et al 2018, dieses Heft). Allerdings hemmt der Verbiss in den untersten Lagen die Verjüngung der Flaumeiche deutlich. Von Verbiss betroffen ist auch die Waldföhre, aber in geringerem Mass (Nussbaumer \& Wohlgemuth 2016).

\section{Besiedlungstypen}

Im Gegensatz zu den vielen Untersuchungen von kleinflächigen Störungen, die durch Stürme entstehen und in denen oft ein direkter Einwuchs von Baumarten vom Rand her festgestellt wird (Kramer et al 2014), liegt mit der grossen Waldbrandfläche von Leuk ein Untersuchungsobjekt vor, das bezüglich Dimension und Störungsintensität in der Schweiz einmalig ist. Die Aspe spielt in diesem Gebiet eine ausgesprochen prominente Rolle, denn sie war nicht nur die erste Baumart, die sich im Gebiet etablierte, sondern sie eroberte mit ihren Ausläufern und Wurzelbruten grössere unbestockte Flächen und nutzte den anfänglichen Freiraum und die ausgezeichnete Nährstoffsituation am besten von allen Baumarten. Wegen der Ausläufer entspricht die Stammzahl nur zu einem Bruchteil der Anzahl Individuen. Angesichts der aktuellen Dominanz kann von einer längeren «Aspenphase» ausgegangen werden. Ebenfalls von Beginn an erfolgreich waren die Pioniere Weide und Birke, die mit ihren kleinen, flugfähigen Samen die Brandfläche von unten bis oben besiedelten. Eine Regenerationsphase mit Aspe, Birke- und Weidenarten, wie sie in der Brandfläche grossflächig besteht, ist typisch in östlich von der Schweiz gelegenen Mittelgebirgen, zum Beispiel im Šumava-Nationalpark in der Tschechischen Republik (Jonášová \& Prach 2004), oder auch im TieflandUrwald Białowieża in Polen (Bernadzki et al 1998). Man darf gespannt sein, wie lange die Vorherrschaft der Aspe dauern wird. Es fragt sich deshalb, wann die Schlussbaumarten in dieser grossen Fläche wieder Überhand nehmen werden und ob dabei die drei Pionierbaumarten eine fördernde Rolle (engl. facilitation) spielen werden. Zehn Jahre nach dem Feuer herrschen jene Baumarten vor, die zuerst keimten oder austrieben (Flaumeiche). Dieser Befund würde das Konzept der Artenzusammensetzung der Ausgangsvegetation (Egler 1954) stützen. Davon ausgehend rechnen wir damit, dass in absehbarer Zeit ab einer Höhe von rund $1300 \mathrm{~m}$ ü. M. ein dichter Baumbestand entstehen wird, dass aber Jahrzehnte, wenn nicht ein bis zwei Jahrhunderte vergehen werden, bis ein Wald nachgewachsen sein wird, der dem Ursprungszustand vor dem Brand entspricht. In Lagen von 900 bis $1300 \mathrm{~m}$ ü.M. wird die Wiederbewaldung auch, aber langsamer, voranschreiten. Dieser Waldbrand wird also lange sichtbar bleiben.

Eingereicht: 6. April 2018, akzeptiert (mit Review): 5. Juli 2018

\section{Dank}

Für die Beteiligung an den Feldarbeiten 2004 bis 2013 danken wir Sara Bangeter, Alexandra Bunge, Anda Fescenko, Stefan Hadorn, Tabea Kipfer, Sarah Kreuzer, Marlen Kube, Salome Leugger, Heiko Schindler, Lukas Wohlgemuth und Martin Zbinden. Ein Dank für den technischen Support geht an Claudio Cattaneo, Dieter Trummer und Ulrich Wasem. Während der ganzen Zeit durften wir vom grossen Entgegenkommen von Forst Region Leuk und der Burgergemeinde Leuk, der Waldbesitzerin, profitieren. Das Projekt wurde von der Dienststelle Wald und Landschaft des Kantons Wallis und dem WSL-Programm Walddynamik finanziell unterstützt.

\section{Literatur}

ALLEN CD, BRESHEARS DD, MCDOWELL NG (2015) On underestimation of global vulnerability to tree mortality and forest die-off from hotter drought in the Anthropocene. Ecosphere 6: 1-55.

BALTISBERGER M, NYFFELER R, WIDMER A (2013) Systematische Botanik: Einheimische Farn- und Samenpflanzen. Zürich: VDF Hochschulverlag, 4 ed. 378 p.

BARBÉRO M, LOISEL R, QUÉZEL P, RICHARDSON DM, ROMANE F (1998) Pines of the Mediterranean basin. In: Richardson DM, editor. Ecology and biogeography of Pinus. Cambridge: Cambridge Univ Press. pp. 450-473. 
BERNADZKI E, BOLIBOK L, BRZEZIECKI B, ZAJACZKOWSKI J, ŻYBURA H (1998) Compositional dynamics of natural forests in the Bialowieza National Park, northeastern Poland. J Veg Sci 9: 229-238.

CAPITANIO R, CARCAILLET C (2008) Post-fire Mediterranean vegetation dynamics and diversity: A discussion of succession models. For Ecol Manage 255: 431-439.

CONNELL JH, SLATYER RO (1977) Mechanisms of succession in natural communities and their role in community stability and organization. Am Nat 111: 1119-1144.

DELARZE R, CALDELARI D, HAINARD P (1992) Effects of fire on forest dynamics in southern Switzerland. J Veg Sci 3: 55-60.

DELARZE R, WERNER P (1985) Evolution après des incendies d'une pelouse steppique et d'une pinède dans une vallée intra-alpine (Valais Central). Phytocoenlogia 13: 305-321.

DONATO DC, HARVEY BJ, TURNER MG (2016) Regeneration of montane forests 24 years after the 1988 Yellowstone fires: A fire-catalyzed shift in lower treelines? Ecosphere 7: e01410.

EGLER FE (1954) Vegetation science concepts. 1. Initial floristic composition, a factor in oldfield vegetation development. Vegetatio 4: 412-417.

FONTURBÉL MT, VEGA JA, PÉREZ-GOROSTIAGA P, FERNÁNDEZ C, ALONSO M ET AL (2011) Effects of soil burn severity on germination and initial establishment of maritime pine seedlings, under greenhouse conditions, in two contrasting experimentally burned soils. Int J Wildl Fire 20: 209-222.

GILL AM (1997) Eucalypts and fire: interdependent or independent. In: Williams JE, Woinarski J, editors. Eucalypt ecology: individuals to ecosystems. Cambridge: Cambridge Univ Press. pp. 151-167.

GÖDICKEMEIER I (1998) Analyse des Vegetationsmusters eines zentralalpinen Bergwaldgebiets. Zürich: ETH Zürich, Dissertation 12641. $127 \mathrm{p}$.

GÓMEZ-GONZÁLEZ S, TORRES-DÍAZ C, BUSTOS-SCHINDLER C, GIANOLI E (2011) Anthropogenic fire drives the evolution of seed traits. Proc Natl Acad Sci USA 108: 18743-18747.

HARVEY BJ, DONATO DC, TURNER MG (2016) Drivers and trends in landscape patterns of stand-replacing fire in forests of the US Northern Rocky Mountains (1984-2010). Landsc Ecol. 31: 2367-2383.

HARVEY BJ, HOLZMAN BA (2014) Divergent successional pathways of stand development following fire in a California closed-cone pine forest. J Veg Sci 25: 88-99.

JOHNSTONE JF, KASISCHKE ES (2005) Stand-level effects of soil burn severity on postfire regeneration in a recently burned black spruce forest. Can J For Res 35: 2151-2163.

JONÁšOVÁ M, PRACH K (2004) Central-European mountain spruce (Picea abies [L.] Karst.) forests: regeneration of tree species after a bark beetle outbreak. Ecol Eng 23: 15-27.

KEELEY JE, BRENNAN T, PFAFF AH (2008) Fire severity and ecosystem responses following crown fires in California shrublands. Ecol Appl 18: 1530-1546.

KEELEY JE, PAUSAS JG, RUNDEL PW, BOND WJ, BRADSTOCK RA (2011) Fire as an evolutionary pressure shaping plant traits. Trends Plant Sci 16: 406-411.

KRAMERK, BRANG P, BACHOFEN H, BUGMANN H, WOHLGEMUTHT (2014) Site factors are more important than salvage logging for tree regeneration after wind disturbance in Central European forests. For Ecol Manage 331: 116-128.

KÜTTEL P (2004) Vegetationswechsel nach Waldbrand in Leuk. Wädenswil: Zürcher Hochschule Angewandte Wissenschaften, Diplomarbeit. $37 \mathrm{p}$.

LAUBER K, WAGNER G, GYGAX A (2012) Flora Helvetica. Bern: Haupt, 5 ed. 1946 p.

LONDO G (1975) Dezimalskala für die vegetationskundliche Aufnahme von Dauerquadraten. In: Schmidt W, editor. Sukzessionsforschung. Ber Int Symp Rinteln 1973. pp. 316-317.
MAGNIER C, TRÉGOUËT B (2011) Le risque de feux de forêts en France. Paris: Conseil général de l'environnement et du développement durable (CGEDD), Etudes et Documents 45. $44 \mathrm{p}$.

MARINGER J, CONEDERA M, ASCOLI D, SCHMATZ D, WOHLGEMUTH T (2016) Resilience of European beech forests (Fagus sylvatica L.) after fire in a global change context. Int J Wild Fire 25: 699-710.

MAUMARY L, VALLOTTON L, DELARZE R (1995) Evolution après incendie d'une pinède et d'une steppe dans une vallée intra-alpine (Valais central). Phytocoenologia 25: 305-316.

MORETTI M, WERMELINGER B, GOSSNER MM, OBRIST MK (2018) Wiederbesiedlung der Waldbrandfläche von Leuk durch Gliederfüsser. Schweiz Z Forstwes 169: 290-298. doi: 10.3188/ szf.2018.0290

MOSER B, GIMMI U, WOHLGEMUTH T (2006) Ausbreitung des Erdbeerspinats Blitum virgatum nach dem Waldbrand von Leuk, Wallis (2003). Bot Helv 116: 179-183.

MOSER B, TEMPERLI C, SCHNEITER G, WOHLGEMUTH T (2010) Potential shift in tree species composition after interaction of fire and drought in the central Alps. Eur J For Res 129: 625-633.

NUSSBAUMER C, WOHLGEMUTH T (2016) Verbiss bremst junge Flaumeichen. Wald Holz 97 (1): 31-33.

R DEVELOPMENT CORE TEAM (2016) R: A language and environment for statistical computing. Vienna: R Foundation for Statistical Computing.

ROMME WH, BOYCE MS, GRESSWELL R, MERRILL EH, MINSHALL GW ET AL (2011) Twenty years after the 1988 Yellowstone fires: Lessons about disturbance and ecosystems. Ecosystems 14: 1196-1215.

SASS O, SARCLETTI S (2017) Patterns of long-term regeneration of forest fire slopes in the Northern European Alps - a logistic regression approach. Geogr Ann Ser A-Phys Geogr 99: 56-71.

SERENA M (2005) Ökologische Resilienz zwei Jahre nach dem Waldbrand in Leuk. Wädenswil: Zürcher Hochschule Angewandte Wissenschaften, Diplomarbeit. 57 p.

TEMPERLI C (2007) Vegetation dynamics after forest fire in comparison to the pre-fire state. Zürich: ETH Zürich, MSc Thesis. $90 \mathrm{p}$.

TURNER MG, ROMME WH, GARDNER RH, HARGROVE WW (1997) Effects of fire size and pattern on early succession in Yellowstone National Park. Ecol Monogr 67: 411-433.

VACCHIANO G, STANCHI S, MARINARI G, ASCOLI D, ZANINI E ET AL (2014) Fire severity, residuals and soil legacies affect regeneration of Scots pine in the Southern Alps. Sci Total Environ 472: 778-788.

VACIK H, ARNDT N, ARPACI A, KOCH V, MÜLLER M ET AL (2011) Characterisation of forest fires in Austria. Austrian J For Sci 128: 1-31.

WERLEN C (1995) Spécificités de la végétation des forêts du Valais. Saussurea 26: 29-35.

WHITE PS, JENTSCH A (2001) The search for generality in studies of disturbance and ecosystem dynamics. Prog Bot 62: 399-449.

WILSON JB, GITAY H, ROXBURGH SH, KING WM, TANGNEY RS (1992) Egler's concept of initial floristic composition in succession - Ecologists citing it don't agree what it means. Oikos 64: 591-593.

WOHLGEMUTH T, BRIGGER A, GEROLD P, LARANJEIRO L, MORETTI M ET AL (2010) Leben mit Waldbrand. Birmensdorf: Eidgenöss Forsch.anstalt WSL, Merkbl Prax 46. 16 p.

WOHLGEMUTH T, DOUBLET V, NUSSBAUMER C, FEICHTINGER L, RIGLING A (2018) Baumartenwechsel in den Walliser Waldföhrenwäldern verstärkt nach grossen Störungen. Schweiz Z Forstwes 169: 260-268. doi: 10.3188/szf.2018.0260

WOHLGEMUTH T, DUELLI P, GINZLER C, GÖDICKEMEIER I, HADORN P ET AL (2005) Ökologische Resilienz nach Feuer: Die Waldbrandfläche Leuk als Modellfall. Schweiz Z Forstwes 156: 345-352. doi: 10.3188/szf.2005.0345 
WOHLGEMUTH T, KRAMER K (2015) Waldverjüngung und Totholz in Sturmflächen 10 und 20 Jahre nach Lothar (1999) und Vivian (1990). Schweiz Z Forstwes 166: 135-146. doi: 10.3188/ szf. 2015.0135

WOHLGEMUTH T, MOSER B (2009) Phönix aus der Asche. Die rasche Wiederbesiedlung der Waldbrandfläche oberhalb von Leuk durch Pflanzen. Bull Murith 126: 29-46.

\section{Dix années de dynamique de végétation sur la zone incendiée de Loèche (Valais)}

L'incendie de la forêt de Loèche (2003) a sévi de 900 à 2100 m d'altitude et a représenté, sur une surface de 300 ha, un évènement perturbateur important pour la Suisse, même s'il reste mineur à l'échelle mondiale. Sa propagation le long d'un gradient d'altitude offre une occasion unique d'étudier l'évolution de la diversité des essences et le rajeunissement naturel sur une grande surface en fonction de différents facteurs. Pendant dix ans, l'évolution de la végétation sur la zone incendiée a été suivie sur 148 parcelles d'échantillonnage et cinq périodes (2004, 2005, 2006, 2007 et 2013). Les résultats montrent que la diversité des espèces a atteint un maximum lors de la quatrième année de prélèvement, et surpassait largement à cette époque celle que l'on observe dans des forêts non perturbées. Là où le feu a été particulièrement violent, la diversité des espèces a moins progressé au cours des quatre premières années que sur les lieux où son intensité a été moindre. Au-dessus d'environ 1600 m, cette diversité a augmenté nettement en raison du nombre plus important d'espèces de montagne. La reforestation progresse à toutes les altitudes. Le nombre d'arbres d'une hauteur supérieure à $25 \mathrm{~cm}, 1800$ à 3000 par hectare, se situe à un niveau plutôt faible dix ans après l'incendie. Les trembles (Populus tremula), saules (Salix appendiculata et $S$. caprea) et bouleaux (Betula pendula) sont les plus nombreux, surtout à des altitudes inférieures à $1700 \mathrm{~m}$, où le rajeunissement est constitué à $95 \%$ de feuillus. Plus haut, on observe des mélèzes ( $L a$ rix decidua) et épicéas (Picea abies), participant chacun à hauteur de $12 \%$ au rajeunissement. En partie basse de la zone incendiée, il s'est établi après dix ans plus de chênes (Quercus pubescens; 6.4\%) que de pins sylvestres (Pinus sylvestris; $3.7 \%)$, mais les proportions de ces essences qui constituaient les peuplements antérieurs sont encore faibles, avec $10 \% \mathrm{du}$ nombre total de troncs. Ainsi, la jeune forêt qui s'établit sur la zone incendiée est constituée à nouveau en partie haute d'épicéas et de mélèzes, et en partie basse d'un peuplement mixte à forte proportion de chênes. On observera si résineux et chênes pubescents y remplaceront trembles, saules et bouleaux, actuellement prédominants, et si c'est le cas, à quelle vitesse.
WOHLGEMUTH T, SCHWITTER R, BEBI P, SUTTER F, BRANG P (2017) Post-windthrow management in protection forests of the Swiss Alps. Eur J For Res 136: 1029-1040.

ZUMBRUNNEN T, BUGMANN H, CONEDERA M, BÜRGI M (2009) Linking forest fire regimes and climate: $A$ historical analysis in a dry inner Alpine valley. Ecosystems 12: 73-86.

\section{Ten years of vegetation dynamics in a forest fire patch in Leuk (Valais)}

The stand-replacing forest fire of Leuk (2003) extends from 900 to $2100 \mathrm{~m}$ a.s.l. and covers an area of 300 ha, which represents a big disturbance event in Switzerland, although it is small in a global perspective. Its location along a wide elevational gradient has offered a unique opportunity to study the dynamics of plant species richness and natural tree regeneration in relation to different environmental drivers. The vegetation was assessed on a systematic grid including 148 relevé plots and 5 time steps. Results show a peak of species richness in the fourth year after the disturbance and a distinctly higher number of species compared to the pre-fire forests. The increase of species richness during the first four years was slower on plots with highest fire severity. Above $1600 \mathrm{~m}$ a.s.l. species richness rose quickly above average due to the appearance of various mountain species. Tree regeneration has advanced in all studied elevation belts. Ten years after the disturbance, sapling densities $\geq 25 \mathrm{~cm}$ reached a rather low level of 1800 to 3000 stems per hectare. Aspen (Populus tremula), willow (Salix appendiculata and S. caprea), as well as birch (Betula pendula) are most abundant up to $1700 \mathrm{~m}$ a.s.l., where up to $95 \%$ of the regeneration consists of broadleaves. Above, European larch (Larix decidua) and Norway spruce (Picea abies) each constitute $12 \%$ of the regenerating saplings. At lowest elevations, oaks (Quercus pubescens) have established more frequently (6.4\%) after ten years than Scots pine (Pinus sylvestris; $3.7 \%$ ), even though the proportion of these formerly stand-forming tree species is still quite modest compared to the total number of stems per ha. Looking ahead, a young forest will grow on the forest fire patch, developing towards a larch-spruce forest at high elevations and towards a mixed forest with a considerable proportion of oak at low elevations. It may take quite a while until prevailing aspen, willow and birch will be overgrown by conifers and oaks. 